\title{
Anterior Knee Pain Syndrome. A Review of Current Concepts and Controversies
}

\author{
Lt Col JB Hull \\ MD, FRCS(Orth), RAMC \\ Consultant Orthopaedic Surgeon
}

\author{
Maj C Hobbs \\ FRCS, RAMC \\ Orthopaedic Specialist Registrar \\ MDHU Frimley Park Hospital, Portsmouth Road, Frimley , Surrey GU16 5UJ
}

\section{Capt S Sidebottom \\ SRP, RAMC \\ Senior Physiotherapist}

DSMRU, Headley Court, Leatherhead, Surrey.

SUMMARY: Anterior knee pain syndrome is a common condition in the military population. Current management is moving away from surgery as the most effective treatment, towards prolonged specific physiotherapy to recondition the extensor mechanism, especially the Vastus Medialis Obliquus (VMO) component of the quadriceps.

\section{Introduction}

The clinical descendant of chondromalacia patellae, Anterior Knee Pain Syndrome (AKPS), continues to comprise a large proportion of military orthopaedic outpatient diagnoses. In the author's own practice, at least half of all new referrals are AKPS, the majority of which are of long-standing duration and are thus leading to employment difficulties. Around $25 \%$ of recruit medical discharges are known to be due to AKPS, most of which are not referred to an orthopaedic consultant. AKPS is endemic in the young military population and is also frequently encountered in the equivalent civilian population, particularly in those who aspire to sporting success.

It remains incompletely understood why some individuals experience AKPS. In the past, considerable attention has been paid to patellofemoral (PF) maltracking as a cause of symptoms but it appears that this anatomical basis for the syndrome is present in only a minority of cases. Current research to define the true prevalence of PF tracking "abnormalities" and attempting to correlate these with symptoms, is on-going. The majority of AKPS patients would appear to have normal PF tracking and thus attempts to alter this surgically are unlikely to be successful (1).

There seems little doubt that surgery is not the solution for the majority of patients. Unfortunately this lesson has been slowly learnt at the expense of many normal joints, and the question of how best to identify those few patients with true maltracking remains difficult to answer. The paper will attempt to define the problems, describe current investigations and treatment, and suggest algorithms for treatment at both primary and secondary level. Although written with a military population in mind, the principles read across well to civilian practice.

\section{Definition of terms}

There is a need to define what is meant by AKPS. It is a syndrome comprising usually intermittent pain deep to or around the margins of the patella. The pain is usually exacerbated by exercise, especially high impact activities such as running or squash. The pain is frequently described as a burning ache, not dissimilar to toothache. There is often associated stiffness in the knee but no true locking or instability. The onset of symptoms is generally gradual although it may be attributed to a specific injury for the reasons given below. As for the site of origin of the pain, this is variable but generally it is either the PF joint itself or its immediate soft tissue relations. These do not include the patellar tendon which, in clinical terms, is a separate entity and so-called patellar tendonosis should be regarded as a separate discrete conditon.

AKPS is not a diagnosis of exclusion. Although other knee injuries or conditions must be excluded, AKPS is a definite diagnosis albeit with a broad spectrum of severity. It is especially important to differentiate AKPS from osteoarthritis of the PF joint, as the management and particularly the prognosis is very different. The key differentiating factor is that the articular surfaces in AKPS are essentially normal and not degenerate. There is no evidence to suggest that AKPS in the absence of significant maltracking predisposes to OA and it is of fundamental importance that this is well understood by both doctors and patients.

\section{Biophysiological considerations}

The most important concern with AKPS is whether there is a structural abnormality responsible for the symptoms which, if left untreated, will lead to serious sequelae. It is probable that for the majority of patients this is not the case. Maltracking of the $\mathrm{PF}$ joint is rare although the true prevalence in the normal population is unknown. AKPS secondary to injury to the PF joint surfaces is also unusual but may be regarded as the first stage of articular surface degeneration, as should any significant joint injury elsewhere in the body.

If it is accepted that most patients will have no demonstrable abnormality (2), there is a need to define and describe those that do.

\section{Maltracking}

Normal tracking

The normal PF joint comprises patella, femoral trochlea and soft tissue attachments. The latter are the quadriceps insertion, the patellar tendon origin, the retinacula and any synovial plicae. In full extension, the patella lies proximal to the trochlear groove and the retropatellar surface rests on the soft floor of the suprapatellar pouch. Medial-lateral mobility of the patella is usually quite free in this position as it is not engaged in the trochlea. 
As knee flexion commences, the lower pole of the patella begins to articulate with the trochlea and by 20 degrees of flexion should be well engaged into the groove. This engagement should be symmetrical and balanced with equal apposition of both patella facets to the corresponding areas on the trochlear slopes. As flexion proceeds, the patella remains engaged throughout, becoming firmly locked into place by 30 40 degrees.

\section{Subluxation}

If patella engagement fails to be symmetrical, the patella may sublux laterally, partially overriding the lateral femoral condyle. This subluxation may only be very mild, exist only in the first 10-15 degrees of flexion, and by 25 degrees of flexion be no longer present. In its most extreme form, lateral subluxation leads on to frank dislocation with damage to the joint surfaces and rupture of the medial retinaculum. In milder form, subluxation in the low degrees of flexion probably causes AKPS by increased contact pressures on the lateral joint surfaces, and may eventually cause pathological change if uncorrected.

\section{Tilt}

Tilt differs from subluxation in that the patella engages into the trochlea centrally, but fails to lie symmetrically, usually due to tethering of the lateral soft tissues (retinaculum) (3). The medial facet lifts away from the medial trochlear slope and the lateral facet angulates away from its normal position parallel to the lateral slope. A reduction in medial joint contact pressure may be responsible for pain and tenderness felt medially to the patella. Tilt due to tightness of the lateral retinaculum is frequently associated with tenderness in this structure and an incidence of neuroma formation (4). Again, tilt is usually corrected by 25-30 degrees of knee flexion, as patellar engagement progresses.

\section{Tilt-with-subluxation}

Both conditions may be found to co-exist in some patients and clearly the symptoms and potential sequelae will be additive. A tight lateral retinaculum may predispose to subluxation.

\section{Trauma}

Direct trauma to the PF joint is probably the next most frequent of the known causes of AKPS. Characteristically the patient describes an injury involving a direct blow to the front of the knee or a fall onto the patella. This does not need to be particularly severe and even trivial injuries can lead to pain, such is the sensitivity of the articular surfaces in some individuals. These patients often have demonstrable surface damage seen on MRI or at arthroscopy and it is not difficult to appreciate why they suffer the symptoms of AKPS. Knee injuries, and surgical interventions, that do not directly involve the PF joint may also lead to AKPS for reasons described below in discussion of the concept of patellofemoral balance.

\section{Malrotation}

The normal relationship between the patella and femur is dependent on normal developmental rotation of the whole lower extremity. Excessive femoral anteversion and excessive tibial external rotation will both lead to increased lateralising forces on the PF joint, and thus to AKPS (5).

Similarly, over-pronation of the midfoot with compensatory hind foot valgus causes rotation of the tibia during gait. This in turn can predispose to AKPS.

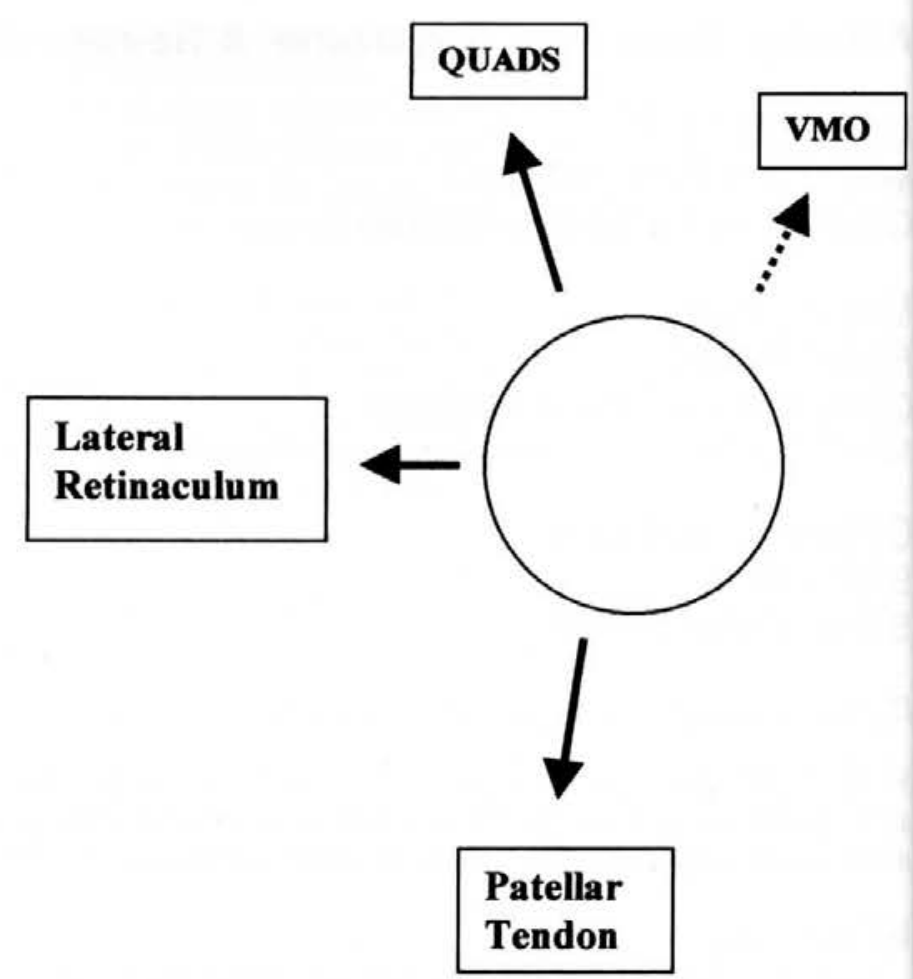

Fig 1. Diagram showing relative soft tissue forces acting on right patella. The VMO opposes the tendency for the patella to track laterally. (VMO: Vastus medialis obliquus)

\section{The normal knee and patellofemoral balance}

Very often, indeed in the majority of patients, no apparent structural abnormality can be demonstrated and there is also no indication that the PF joint is degenerate. That these patients have genuine symptoms is undisputed and it can be frustrating for both physician and sufferer to have no reason for the discomfort.

The concept of patellofemoral balance is worthy of consideration. As stated above, the PF joint is dependent for it's stability on soft tissue attachments. The anatomical arrangement of the quadriceps and patellar tendon is such that there is a lateralising vector acting on the patella as both the Anterior Superior Iliac Spine and tibial tuberosity lie lateral to the PF joint (Fig 1). Structures that counteract this tendency are the medial retinaculum which is largely static and the VMO muscle which is part of the quadriceps and inserts directly into the medial edge of the patella. Relative weakness of VMO allows minor degrees of subluxation to occur during early flexion and contributes to instability. Equally, minor abnormalities such as slight tethering in the iliotibial band which pulls on the lateral retinaculum, may be sufficient to increase lateral joint pressure to the point where symptoms are experienced (6).

This concept certainly helps explain why AKPS develops frequently when individuals suddenly increase their exercise activity levels, such as during basic training or intensive courses. In the majority, symptoms settle with or without rest preumably as the VMO "catches up" and becomes fitter. A similar picture is seen following knee surgery and trauma unrelated to the PF joint, when AKPS is frequent and usually transient.

That the medial dynamic stabilisers of the PF joint are important is quite clear from the expected improvement wth VMO strengthening programmes such as the McConnell regime. Together with patellar taping, this aims to progressively off-load the lateral facet reducing pain and ultimately the minor 
degree of instability of the PF joint in the low angles of flexion (7).

It is not yet clear why some individuals with normal PF joints get recurrent and sometimes disabling symptoms and many others do not. It is probable that we are unable to detect the very minor degrees of imbalance that certain patients are prone to, and which respond badly to increased exercise demands. Whereas in civilian life, these individuals may well avoid strenuous exercise from an early age and not go on to become athletes, military personnel have no choice in the matter and thus AKPS presents commonly.

\section{Clinical Features \\ Pain}

The pain of AKPS is typically burning in nature, often described as a "toothache" in the knee. It is usually intermittent and is made worse by exercise, especially if this involves high impact. Pain at rest after exercise is usual and may be the only time this occurs. The location of the pain is variable but is generally under (deep to) or around the margins of the patella. When asked to locate their pain, many patients lay their open hand over the patella and are unable to localise the site of discomfort better than this; some will quite clearly indicate the lateral retinaculum as the painful area. The lower pole of the patella and upper patellar tendon are common sites of pain and care must be made to differentiate AKPS from localised patellar tendonisis. In severe cases the pain may be extreme and constant, preventing any comfortable ambulation.

Typically, stairs and slopes exacerbate the pain, descent often being more uncomfortable than ascent. This is logical as stability of the PF joint is reduced in the low angles of flexion, more usual during descent. Many patients find they cannot tolerate sitting with their knees bent for long, especially as a car passenger. As flexion increases so does PF joint reaction force, so this too seems logical. Typically, patients need to stop and stretch their legs at intervals during a journey.

\section{Stiffness and pseudo-locking}

Together with pain, there is a sensation of stiffness at the front of the knee which at its extreme can appear to be a form of locking. Unlike true mechanical locking from a displaced bucket handle meniscal tear, this pseudo-locking is due to reflex inhibition of the extensor mechanism caused by pain, and generally the knee frees up after a few minutes of attempting to restart gentle movements. Few patients experience dramatic unlocking and will say that they can get the knee straight eventually.

\section{Pseudo-giving way}

During ambulation, momentary reflex inhibition of the extensor mechanism may result in subjective instability and a feeling that the knee is giving way. Many patients describe a slight buckling of the knee but this is very rarely severe or prolonged enough to make them actually fall over, unlike the true giving way of ligamentous instability. Most individuals feel most at risk of this pseudo-giving way when descending stairs or steep slopes.

\section{Swelling}

AKPS does not produce appreciable knee effusion unless there is significant degenerative change in the knee or an associated mechanical deficit. Patients often describe swelling in the supra or infra-patellar regions but this is never marked. There may be soft tissue thickening especially of the retinacula and this can sometimes be difficult to differentiate from an effusion.

\section{Crepitus}

Crepitus is extremely common as is clicking within the knee. When not painful these clicks are of no significance and certainly do not indicate roughened articular surfaces or loose bodies etc. Many normal knees click and grind and it is important not to attach to much significance to this non-specific finding.

\section{Tenderness}

There may be little or no tenderness around the PF joint when the patient is seen in clinic. At the other extreme, severe AKPS may render the patella untouchable and physical guarding by the patient's hand will be encountered. In most cases, compression and grinding of the joint will be painful and tenderness of the lateral retinaculum is common. Asking the patient to contract quadriceps whilst the joint is compressed (Osmond-Clarke's sign) is almost always very painful and is frequently positive in normal individuals. By performing this manoeuvre it is easy to lose the patient's trust, and as it is nonspecific for AKPS, the author does not recommend its use.

Gently compressing the patella laterally while flexing the knee from 0-30 degrees may reliably reproduce the pain arising from an overstressed lateral facet. By displacing the patella laterally and medially with the knee in full extension, the retropatellar articular surfaces can be examined for tenderness.

Careful examination of the patellar tendon, looking particularly for point tenderness at the proximal end of this structure, is important to exclude the small area of degeneration found in tendonosis.

\section{Movement}

The range of movement is generally not restricted with AKPS. Attempts to fully extend the knee against resistance may be uncomfortable but movement in the range of 30 degrees to full flexion is rarely painful as the patella is fully and symmetrically engaged in the trochlea.

Abnormal movement of the patella during flexion may be observed, although this is not usually helpful in the author's experience and is probably only obvious in extreme cases of maltracking.

\section{Investigations}

\section{Plain radiography}

All patients with significant knee symptoms require plain AP and lateral X-rays to exclude bony defects, loose bodies and early degenerative change. Most will be normal but these serve also as a baseline for the future.

Although some information about the PF joint can be gleaned from the lateral view, particularly whether sclerosis or spur formation indicates degeneration, the view of choice for AKPS is the tangential skyline view. Previously thought to be necessary in 30,60 and 90 degrees of flexion, it appears now that the most important view is that taken at 20 degrees. This is because once the patella has fully engaged, the radiological appearance of the joint is unlikely to yield further useful information. Furthermore, it has been shown that skyline views in the higher angles of flexion are frequently normal despite significant maltracking in the range $0-30$ degrees as shown by CT scan (8).

The 20 degree skyline view is achieved by placing the patient supine with a pillow under the knee creating flexion. The X-ray source is rested on the front of the ankle pointing proximally "through" the PF joint. The patient holds a plate perpendicular to the beam against his upper thigh and the image is captured. A skyline view at less than 20 degrees of flexion is prevented by superimposition of the femur and tibia. 
The 20 degree skyline view gives useful information about the relative positon of the patella and trochlea as well as symmetry of the joint. It is possible to identify both patellar tilt and subluxation, and thus determine whether surgery is warranted to address maltracking. Tilt is assessed by calculation of the patella tilt angle, the angle between the lateral facet and a line joining the most prominent aspects of the femoral trochlea. Subluxation is determined by the sulcus angle which compares the line adjoining the bottom of the trochlea sulcus and the lowest point on the patella with a line bisecting the trochlear sulcus (9). Because the X-ray is taken with the quadriceps relaxed, it is possible that it is not a true reflection of PF alignment during function. A study comparing the 20 degree view with cine MRI findings is on-going.

\section{CT Scan}

In order to overcome the problem of superimposition in the low angles of flexion, CT scans of the PF joint may be useful. Scans at 5, 10, 15 and 20 degrees of flexion yield useful information about tilt and subluxation but necessarily involve considerable radiation exposure and are expensive.

\section{Axial cine-MRI}

If rapidly acquired MR images are made of the PF joint from full extension to 30 degrees of flexion, it is possible to generate a dynamic sequence of PF articulation in the form of a cine loop. This gives clear information about tracking in the low angles and may identify subluxation that is not apparent by other means. In addition, MR gives useful information concerning the condition of the articular cartilage and will identify areas of surface irregularities. MRI is time-consuming and expensive and is probably inappropriate for routine use; it may be helpful for patients who do not improve with standard treatment.

\section{Arthroscopy}

Arthroscopic examination of the PF joint is useful to confirm the state of articular cartilage but is not helpful in the diagnosis of minor degrees of maltracking. By inflating the joint with fluid, the normal relationship of the patella and trochlea is distorted and it is impossible to determine whether tilt or subluxation is present. Scarring and tethering of the lateral retinaculum may be identified, but if significant, this should have been diagnosed by other investigations. Arthroscopy will identify other intra-articular pathology but is only marginally more sensitive than standard MRI

\section{Treatment}

The most important consideration in the management of AKPS is understanding. It is vital that both patient and physician appreciate the nature of the syndrome, especially the likelihood that no discernible abnormality will be discovered by investigation. Another feature of fundamental importance is the low risk of long term sequelae in those with normal joint surfaces and no significant maltracking. Education and reassurance are of great value in treatment and must not be ignored.

\section{Rest}

From the above discussion of PF balance, it will be clear that absolute rest from exercise is not appropriate. On the contrary, increased levels of structured activity are essential to retrain the VMO and achieve balance in the PF joint. Rest from high impact exercise and hill work for a short period may be indicated but the idea of absolute rest should be discouraged.

\section{Physiotherapy}

This is the mainstay of treatment and incorporates re-micrc education and re-training. All patients with significant AKPS car should be referred to a physiotherapist with experience in this re field, and treatment should continue for a minimum of three mirk months with regular supervision.

The aim of physiotherapy is to recondition the PF joint by team strengthening all muscle groups and particularly the VMO. The McConnell regime for VMO strengthening has good results and Malt should always be tried (10). VMO contracts maximally during the last 10-15 degrees of knee extension and therefore resisted the la extension exercises in this range should be prescribed. It may aforo also be helpful to use tape to hold the patella medially during uşing exercises. The hamstrings and iliotibial band also frequently argd la require stretching. Patella mobilisation exercises may free up vơstu retinacular adhesions and scarring; electrotherapy may have a arthro limited beneficial effect on capsular tenderness. It is important patell however, that the physiotherapist does not rely on electronic urafler gizmos to treat AKPS, as serious success is unlikely. The pave patient must appreciate that outcome is entirely dependent on ary es his or her self-motivation and that the highest quality of of po: physiotherapist will be unable to improve symptoms without considerable hard work by the patient.

Also of importance is the understanding that although symptoms may be improved significantly with treatment, it may not be possible to completely abolish all of the pain. The aim should be to reduce symptoms to a level where full active duties can be undertaken by the military patient, and ful sporting activities can be tolerated by the civilian. Most patients will have some definite improvement with correc physiotherapy $(11,12)$.

As well as addressing knee pain in particular, it is sensible to use access to a physiotherapy department to improve genera levels of fitness where possible. Advice and guidance or running technique and footwear are valuable additional benefit of a physiotherapy referral.

\section{Orthotics}

There is little doubt that excessive running in heavy footwea such as military boots exacerbates AKPS. Unfortunately some boot running and marching is of fundamental importance in military training. If impact can be softened by cushione insoles, this must be of benefit whether or not the individual $i$ symptomatic.

For the patient with midfoot over-pronation, moulde orthotics with a medial heel and forefoot raise may b extremely effective (13). The level of the medial posts must bx carefully assessed by a competent therapist and temporary orthotics should be used initially to ensure the appropriat degree of correction is achieved. Time should be given to allov the patient to adjust to the orthotics and it may take severa weeks before benefit is appreciated. The orthotics should b worn in all footwear, not just trainers or boots and more that one pair of definitive inserts will usually be needed.

\section{Surgery}

The role of diagnostic arthroscopy has been discusse Interventions that may be indicated in the treatment of AKP aim to address two separate pathologies:

- articular surface defects

- maltracking

\section{Articular surface defects}

In those cases where direct trauma to the articular surface has resulted in visible damage, attempts to modify this may b warranted. Loose flaps of cartilage can be excised or shaved 
and exposed subchondral bone may be drilled or microfractured. After a dislocation, large osteochondral defects can sometimes be repaired by direct implantation of the retrieved fragment into the defect. Where articular fibrillation is marked there may be benefit from shaving (14) but as the fibrillation generally results from early degeneration, no longterm improvement can realistically be expected.

\section{Maltracking}

The most common intervention to correct lateral tracking is the lateral retinacular release. Formerly undertaken via an open approach, this is now performed under arthroscopic control using a cutting diathermy probe within the knee. The synovium and lateral capsule are divided longitudinally from the level of vastus lateralis distally as far as the anterolateral portal. The arthrotomy runs approximately $1 \mathrm{~cm}$ lateral to the edge of the patella. Once the capsule is breached, a bleb of fluid appears under the skin, holding it away from the diathermy point thus preventing inadvertent skin puncture. Retinacular vessels that are encountered can be diathermied directly, reducing the risk of postoperative haemarthrosis.

Recovery from lateral release may be prolonged, taking several months of hard physiotherapy to achieve a significant improvement on pre-operative pain. Quadriceps retraining is vital and if a lateral release is not followed by a dedicated exercise programme, a poor result is often achieved. Lateral release by necessity reduces patellofemoral stability at first and without quads control, this persists.

It is vital that lateral release be undertaken to correct a definite tracking abnormality such as tilt and/or subluxation. There is no place for lateral release as a last resort in apparently normal knees as it may well result in medial subluxation and instability (15). Clinical studies showing poor results should be assessed critically to identify how well these guidelines were adhered to. Several studies report success rates for lateral release of up to $80 \%(16,17)$. The author's experience of this procedure is that it produces definite improvement in most cases, if one is prepared to wait for up to six months following the operation.

Other operations to correct maltracking involve revising the attachments of the medial stabilising structures. The medial retinaculum can be plicated and VMO can be advanced. All involve open surgery and have very variable results, sometimes improving severe pain but rarely leading to full fitness in the medium or long term.

The more favoured approach at present is to reposition the

Patient presents with knee pain

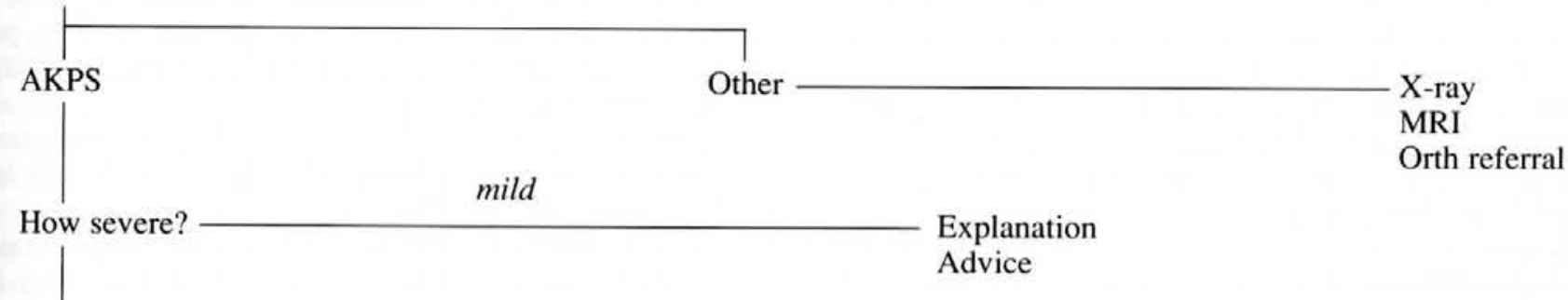

moderate/severe

\section{PHYSIOTHERAPY}

3 months minimum

McConnell / taping

No impact sports
1
improved

3 month reassessment

Return to full duties

worse / no change

ORTHOPAEDIC REFERRAL

Downgrade P3L3 LE[R]

Skyline X-ray / MRI normal

Skyline X-ray / MRI — More physio

Permanent downgrading

maltracking

\section{ARTHROSCOPY}

improved

Lateral release

3-6 months physio

no improvement

Tibial Tuberosity Transfer?

Medical discharge? 
patellar tendon insertion. Simple medialisation is not sufficient and tibial tuberosity transfer must achieve anteriorisation of the tuberosity to be effective by reducing PF joint reaction force (18). Results of these procuedures are variable and it would seem that symptoms have to be really quite severe for the patient to achieve significant improvement. This is certainly not an intervention to consider in the athlete or soldier who has mild to moderate symptoms and who wishes to have none.

Patellectomy used to be a favoured procedure for patellofemoral osteoarthritis and may occasionally dramatically improve function and pain. It is not usually compatible with strenuous physical activity and has been shown to rarely be compatible with continued military service (19). An alternative is patellofemoral arthroplasty, and early results of this are encouraging in the older person.

All procedures beyond lateral release should be regarded very much as second-line salvage manoeuvres and not be offered to patients without very careful consideration.

A suggested management algorithm for AKPS is shown in Figure 2. It demonstrates the central importance of physiotherapy and with the implementation of appropriate exercise regimens the need for orthopaedic referral, and surgery, should be reduced.

\section{REFERENCES}

1. FUlKerson JP, ShEA KP. Current concepts review. Disorders of patellofemoral alignment. J Bone Joint Surg [Am] 1990; 72-A: 1424-1429.

2. Griffiths I, PINDER I. Chondromalacia patellae, a clinical and arthroscopic study. Amn Rheum Dis 1981; 40: 617.

3. Maquet P. Advancement of the tibial tuberosity. Clin Orthop 1976; 115: 225-230.

4. Fulkerson JP, Tennant R, Jaivin JS, Grunnet M. Histologic evidence of retinacular nerve injury associated with patellofemoral malalignment. Clin Orthop 1985; 197: 196-205.

5. Eckhoff DG, Montgomery WK, Kilcoyne RF, Stamm ER. Femoral morphometry and anterior knee pain. Clin Orthop 1994; 302: 64-68.

6. Flatow EL, et al. Computer simulation of glenohumeral and patellofemoral subluxation. Estimating pathologica articular contact. Clin orthop 1994; 306: 28-33.

7. MCCONNELL JS. The management of chondromalaci patellae: a long term solution. Aus J Physiology 1986; 32 215-223.

8. Walker C, Cassar-Pullicino VN, Vaisha R, McCall IW The patello-femoral joint - a critical appraisal of it geometric assessment utilizing conventional axia radiography and computed arthro-tomography. $\mathrm{Br} J$ Radio 1993; 66: 755-761.

9. Carson W, Carson R, Singer K. Patello-femoral disorders physical and radiological evaluaiton. Clin Orthop 1984 185: $165-186$.

10. MCCONNELl JS. Training the Vastus Medialis Oblique in th management of patellofemoral pain. Sydney: Proceeding of the 10th International Congress of the World Federatio of Physical Therapists 1987; Vol 2: 412-415.

11. Dehaven KE, Dolan WA, Mayer PJ. Chondromalaci patellae in athletes. Clinical presentation and conservativ management. Am J Sports Med 1979; 7: 5-11.

12. InSALL J. Current concepts review-patellar pain. $J$ Bon Joint Surg [Am] 1982; 64A: 147-152.

13. ENG JJ, PIERRYNOWSKI MR. Evaluation of soft foot orthotic in the treatment of patellofemoral pain syndrome. Phys The 1993; 73: 62-70.

14. RADIN E. A rational approach to the treatment 0 patellofemoral pain. Clin Orthop 1979; 144: 107-109.

15. Hughston JC, DeEse M. Medial subluxation of the patell as a complication of lateral retinacular release. Am J Sport Med 1988; 16: 383-388.

16. Lankenner P, Micheli L, Çlancy R. Arthroscopi percutaneous lateral patellar retinacular release. Am J Spor Med 1986; 14: 267-269.

17. OgILVIE-HARRIS D. JaCKSON R. The arthroscopic treatmen of chondromalacia patella. J Bone Joint Surg [Am] 1984 66B: $660-665$.

18. RADIN EL. Anterior tibial tubercle elevation in the youn adult. Orthop Clin North Am 1986; 17: 297-302.

19. PAILTHORPE CA, MiLner SM, Sims MM. Is patellectom compatible with an Army career. JR Army Med Corps 199 137:76-79. 